Received: 20 November 2017

Accepted: 18 January 2018

Published online: 02 February 2018

\section{Tunable phonon blockade in quadratically coupled optomechanical systems}

\author{
Hai-Quan Shi' ${ }^{1,2}$, Xiao-Tong Zhou' ${ }^{2}$, Xun-Wei Xu' ${ }^{2}$ \& Nian-Hua Liu ${ }^{1,3}$
}

We theoretically investigate the phonon statistics of a quadratically coupled optomechanical system, in which an effective second-order nonlinear interaction between an optical mode and a mechanical mode is induced by a strong optical driving field on two-phonon red-sideband resonance. We show that strong phonon antibunching can be observed even if the strength of the effective second-order nonlinear interaction is much weaker than the decay rates of the system, by driving the optical and mechanical modes with weak driving fields respectively. Moreover, the phonon statistics can be dynamically controlled by tuning the strengths and the phase difference of the weak driving fields. The scheme proposed here can be used to realize tunable single-phonon sources with quadratically optomechanical coupling.

Phonon blockade ${ }^{1}$, in analogy to the Coulomb blockade ${ }^{2}$, photon blockade ${ }^{3}$ and Rydberg blockaded ${ }^{4-8}$, is a quantum phenomenon that only one phonon can be excited in a nonlinear mechanical oscillator when it is driven by external fields. Phonon blockade has already been studied in a mechanical resonator coupled to a superconducting qubit in the dispersive ${ }^{1,9-11}$ and resonant ${ }^{12,13}$ regimes. Effective phonon-phonon interactions can be induced by the qubit and strong phonon antibunching effect can be observed for large coupling strength and moderate detuning between the mechanical resonator and the qubit.

In the past decades, optomechanical systems have drew great attention in researches on the foundations of quantum theory and quantum information processing (for reviews, see refs ${ }^{14-18}$.). Recently, two different groups studied phonon statistics in quadratically coupled optomechanical systems ${ }^{19,20}$. Seok and Wright found that antibunched single-phonon field appears for large optomechanical cooperativity ${ }^{19}$. Hong Xie et al. found that strong effective phonon-phonon nonlinear interaction as well as phonon blockade can be induced by a strong optical driving field in the quadratically coupled optomechanical system ${ }^{20}$.

In contrast to refs ${ }^{1,9-12,19,20}$, where the phonon blockade is induced by strong effective phonon-phonon interactions, an interference-based phonon blockade called unconventional phonon blockade (UCPNB) was studied in ref. ${ }^{13}$. UCPNB, due to the destructive interference between different paths for two-phonon excitation, can be obtained with weak effective phonon-phonon interactions is similar to the unconventional photon blockade in a weakly nonlinear system of photonic molecule ${ }^{21-33}$. More recently, UCPNB was studies in a coupled nonlinear mechanical system with weak nonlinearity ${ }^{34}$.

In this paper, we shall theoretically investigate UCPNB in a quadratically coupled optomechanical system. An effective second-order nonlinear interaction between an optical mode and a mechanical mode can be induced when the quadratically coupled optomechanical system is driven by a strong optical driving field on two-phonon red-sideband resonance. Beside the strong optical driving field, the optical and mechanical modes are also driven by a weak optical and mechanical fields respectively. Different from the previous studies ${ }^{19,20}$, we will show that strong phonon antibunching can be observed even if the strength of the effective second-order nonlinear interaction is much weaker than the decay rates of the system. Moreover, the phonon statistics can be dynamically controlled by tuning the strengths and the phase difference of the weak driving fields. The proposal provides a simple way to realize tunable single-phonon sources with quadratically optomechanical coupling.

\footnotetext{
${ }^{1}$ School of Materials Science and Engineering, Nanchang University, Nanchang, 330031, China. ${ }^{2}$ Department of Applied Physics, East China Jiaotong University, Nanchang, 330013, China. ${ }^{3}$ Institute for Advanced Study, Nanchang University, Nanchang, 330031, China. Correspondence and requests for materials should be addressed to N.-H.L. (email:nhliu@ncu.edu.cn)
} 


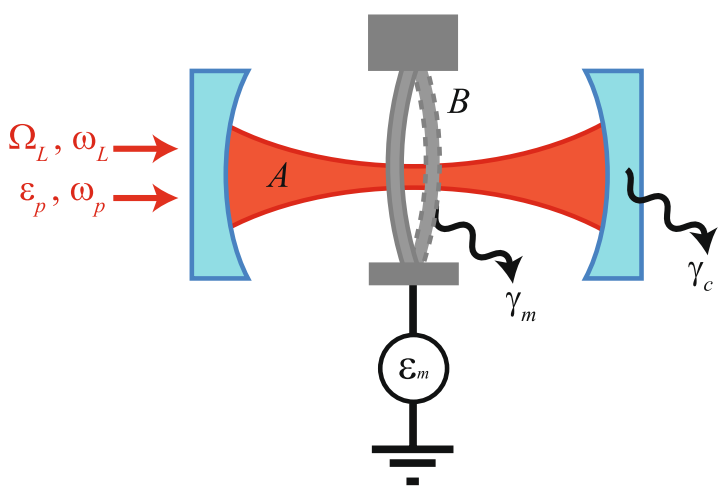

Figure 1. The schematic sketch of a quadratically coupled optomechanical system.

\section{Results}

Theoretical model and analytical results. We study a quadratically coupled optomechanical system in which an optical mode is coupled to the second order of the position of a mechanical mode, as schematically shown in Fig. 1. The optical mode with frequency $\omega_{c}$ is driven by a strong driving field with the strength $\left|\Omega_{L}\right| \gg\left\{\gamma_{c}, \gamma_{m}\right\}$ and frequency $\omega_{L}$, where $\gamma_{c}$ and $\gamma_{m}$ are the damping rates of the optical and mechanical modes and $\Delta_{c} \equiv \omega_{c}-\omega_{L}$ is the frequency detuning between the strong driving field and the optical mode. Meanwhile, the optical mode and mechanical mode (frequency $\omega_{m}$ ) are driven by weak external fields with strengths $\left\{\left|\varepsilon_{p}\right|\right.$, $\left.\left|\varepsilon_{m}\right|\right\}<\left\{\gamma_{c}, \gamma_{m}\right\}$ and frequencies $\left\{\omega_{p}, \omega_{d}\right\}$, with the detuning between the optical driving fields $\delta_{p}=\omega_{p}-\omega_{L}$. The Hamiltonian for quadratically coupled optomechanical system in the rotating reference frame with optical frequency $\omega_{L}$ takes the form $(\hbar=1)$

$$
\begin{aligned}
H= & \Delta_{c} A^{\dagger} A+\omega_{m} B^{\dagger} B+g A^{\dagger} A\left(B^{\dagger}+B\right)^{2} \\
& +\left(\Omega_{L} A^{\dagger}+\varepsilon_{p} e^{-i \delta_{p} t} A^{\dagger}+\varepsilon_{m} e^{-i \omega_{d} t} B^{\dagger}+\text { Hc. }\right),
\end{aligned}
$$

where $A$ and $A^{\dagger}\left(B\right.$ and $B^{\dagger}$ ) denote the annihilation and creation operators for the optical mode (mechanical mode), $g>0$ describes the strength of the quadratic optomechanical coupling between the optical and mechanical modes, and H.c. stands for Hermitian conjugate. The quadratically optomechanical coupling can be found in the optomechanical crystals ${ }^{35}$, Fabry-Perot cavities with membrane-in-the-middle ${ }^{36-39}$, and some other optomechanical systems ${ }^{40-43}$.

The operators can be rewritten as the sum of their steady-state mean values and quantum fluctuation operators as: $A \rightarrow \alpha+a$ and $B \rightarrow \beta+b$, where $\alpha$ and $\beta$ are the steady-state mean values, $a$ and $b$ are the quantum flucturation operators. The steady-state mean values $\alpha$ and $\beta$ can be obtained approximately by setting the strength of the weak driving fields as zero, i.e. $\varepsilon_{m}=\varepsilon_{p}=0$, then we have

$$
\begin{gathered}
\alpha=\frac{-i 2 \Omega_{L}}{\gamma_{c}+i 2 \Delta_{c}}, \\
\beta=0 .
\end{gathered}
$$

After some standard procedures for operator linearization, the Hamiltonian for the quantum flucturation operators reads

$$
\begin{aligned}
H^{\prime}= & \Delta_{c} a^{\dagger} a+\omega_{m} b^{\dagger} b+g\left(|\alpha|^{2}+a^{\dagger} a\right)\left(b^{\dagger}+b\right)^{2} \\
& +g\left(\alpha a^{\dagger}+\alpha^{*} a\right)\left(b^{\dagger}+b\right)^{2} \\
& +\left(\varepsilon_{p} e^{-i \delta_{p} t} a^{\dagger}+\varepsilon_{m} e^{-i \omega_{d} t} b^{\dagger}+\text { Hc. }\right) .
\end{aligned}
$$

For a strong optical driving field $\left|\Omega_{L}\right| \gg\left\{\gamma_{c}, \gamma_{m}\right\}$, we assume that the steady-state mean value $\alpha$ is much larger than the quantum flucturation operators $a$ as $|\alpha|^{2} \gg\left\langle a^{\dagger} a\right\rangle$, so the term $g a^{\dagger} a\left(b^{\dagger}+b\right)^{2}$ in the above equation can be neglected. In the rotating reference frame with respect to the unitary operator $R(t)=\exp \left(i \delta_{p} a^{\dagger} a t+i \omega_{d} b^{\dagger} b t\right)$, the effective Hamiltonian can be obtained under the rotating-wave approximation by neglecting the terms oscillating with high frequencies in equation (4), e.g. $2 \omega_{d}$ and $\delta_{p}+2 \omega_{d}$, as

$$
\begin{aligned}
H^{\prime}{ }_{\text {eff }}= & \Delta_{p} a^{\dagger} a+\Delta_{m} b^{\dagger} b+J\left(e^{-i \theta} e^{-i \delta t} a b^{\dagger 2}+e^{i \theta} e^{i \delta t} a^{\dagger} b^{2}\right) \\
& +\left(\varepsilon_{p} a^{\dagger}+\varepsilon_{m} b^{\dagger}+\text { H.c. }\right),
\end{aligned}
$$

where the detunings $\delta=\delta_{p}-2 \omega_{d}, \Delta_{p}=\Delta_{c}-\delta_{p}, \Delta_{m}=\omega_{m}+2 g|\alpha|^{2}+g-\omega_{d}$, and we assume that the detunings satisfy the condition $\left\{|\delta|,\left|\Delta_{p}\right|,\left|\Delta_{m}\right|\right\} \ll\left\{\omega_{m}, \omega_{d}\right\} . J=g \alpha$ is the effective nonlinear coupling strength between the optical and mechanical modes. Without loss of generality, $J, \varepsilon_{p}$ and $\varepsilon_{m}$ are assumed to be real and the phase 
difference between the driving fields is denoted by $\theta$. For simplicity, we set $\delta_{p}=2 \omega_{d}$ and $\Delta_{c}=2\left(\omega_{m}+2 g|\alpha|^{2}+g\right)$, then we have $\delta=0$ and $\Delta \equiv \Delta_{m}=\Delta_{p} / 2$, and the effective Hamiltonian $H_{\text {eff }}^{\prime}$ become time-independent as

$$
\begin{aligned}
H_{\text {eff }}= & 2 \Delta a^{\dagger} a+\Delta b^{\dagger} b+J\left(e^{-i \theta} a b^{\dagger 2}+e^{i \theta} a^{\dagger} b^{2}\right) \\
& +\left(\varepsilon_{p} a^{\dagger}+\varepsilon_{m} b^{\dagger}+\text { H.c. }\right) .
\end{aligned}
$$

To quantify the statistics of the phonons in the system, we consider the second-order correlation functions in the steady state defined by

$$
g_{b}^{(2)}(\tau) \equiv \frac{\left\langle b^{\dagger}(t) b^{\dagger}(t+\tau) b(t+\tau) b(t)\right\rangle}{n_{b}^{2}},
$$

where $n_{b} \equiv\left\langle b^{\dagger}(t) b(t)\right\rangle$ is the mean phonon number. The dynamic behavior of the total open system is described by the master equation for the density matrix $\rho^{44}$

$$
\begin{aligned}
\frac{d \rho}{d t}= & -i\left[H_{\mathrm{eff}}, \rho\right]+\frac{\gamma_{c}}{2}\left(2 a \rho a^{\dagger}-a^{\dagger} a \rho-\rho a^{\dagger} a\right) \\
& +\frac{\gamma_{m}}{2}\left(2 b \rho b^{\dagger}-b^{\dagger} b \rho-\rho b^{\dagger} b\right) \\
& +\gamma_{m} n_{\mathrm{th}}\left(b \rho b^{\dagger}+b^{\dagger} \rho b-b^{\dagger} b \rho-\rho b b^{\dagger}\right),
\end{aligned}
$$

where we assume that the mean thermal photon number is negligible for the frequency of the optical mode is very high, and $n_{\text {th }}$ is the mean number of the thermal phonons, given by the Bose-Einstein statistics $n_{\mathrm{th}}=\left[\exp \left(\hbar \omega_{m} / k_{B} T\right)-1\right]^{-1}$ with the Boltzmann constant $k_{B}$ and the environmental temperature $T$. The second-order correlation function $g_{b}^{(2)}(\tau)$ can be calculated by solving the master equation (8) numerically within a truncated Fock space.

It is instructive to find the optimal conditions for strong phonon antibunching before the numerical calculations of the second-order correlation function of the phonons. Following the approach given in ref. ${ }^{22}$, the optimal conditions for UCPNB can be derived analytically with the effective Hamiltonian $H_{\text {eff }}$ given in equation (6), in the limit $T \rightarrow 0$ and the weak driving condition $\left\{\varepsilon_{p}, \varepsilon_{m}\right\} \ll\left\{\gamma_{c}, \gamma_{m}\right\}$. The derivation of the the optimal conditions is provided in the section of Methods. When $\theta=N \pi$ ( $N$ is an integer), the optimal conditions are simply given by

$$
\begin{gathered}
\Delta_{\mathrm{opt}}=0, \\
J_{\mathrm{opt}}=-\frac{\varepsilon_{m}^{2} \gamma_{c}}{\varepsilon_{p} \gamma_{m} \cos \theta} .
\end{gathered}
$$

When $\theta \neq N \pi$, the optimal conditions become

$$
\begin{gathered}
\Delta_{\mathrm{opt}}=\frac{\left(\gamma_{c}-2 \gamma_{m}\right) \cos \theta \pm \sqrt{\Psi}}{8 \sin \theta}, \\
J_{\mathrm{opt}}=-\frac{\varepsilon_{m}^{2}}{\varepsilon_{p}} \frac{4 \gamma_{c}}{\left(\gamma_{c}+2 \gamma_{m}\right) \cos \theta \pm \sqrt{\Psi}},
\end{gathered}
$$

where

$$
\Psi=\left(2 \gamma_{m}-\gamma_{c}\right)^{2} \cos ^{2} \theta-8 \gamma_{c} \gamma_{m} \sin ^{2} \theta
$$

In order to make sure that $\Delta_{\text {opt }}$ and $J_{\text {opt }}$ given in equations (11) and (12) have real solutions, the phase $\theta$ should satisfy the condition

$$
|\theta-N \pi| \leq \tan ^{-1}\left(\sqrt{\frac{\left(2 \gamma_{m}-\gamma_{c}\right)^{2}}{8 \gamma_{c} \gamma_{m}}}\right) .
$$

We take $J_{\text {opt }}>0$ in the following numerical calculations, so that $N$ should be an odd number. Without loss of generality, we choose $N=1$.

Numerical results. In order to confirm the appearing of optimal UCPNB with the optimal parameters given in equations (9-13), we numerically solve the master equation (8) and calculate the second-order correlation functions $g_{b}^{(2)}(\tau)$. In Fig. 2(a), the equal-time second-order correlation functions $g_{b}^{(2)}(0)$ is plotted as a function of the detuning $\Delta / \gamma_{c}$ with the effective coupling strength $J=0.025 \gamma_{c}$ and phase $\theta=\pi$. It is clear that the optimal phonon blockade appears at the detuning $\Delta=0$ and this agrees well with the analytical result given in equation (9). The corresponding mean phonon number $n_{b}$ is plotted in Fig. 2(b). The maximal value of $n_{b}$ also appears at the detuning $\Delta=0$ for resonant driving. The dependence of $g_{b}^{(2)}(0)$ on the strength of the effective coupling $J / \gamma_{c}$ is shown in Fig. 2(c) for $\Delta=0$ and $\theta=\pi$. There is a minimal value of $g_{b}^{(2)}(0)$ around $J \approx 0.025 \gamma_{c}$ which is in agree- 

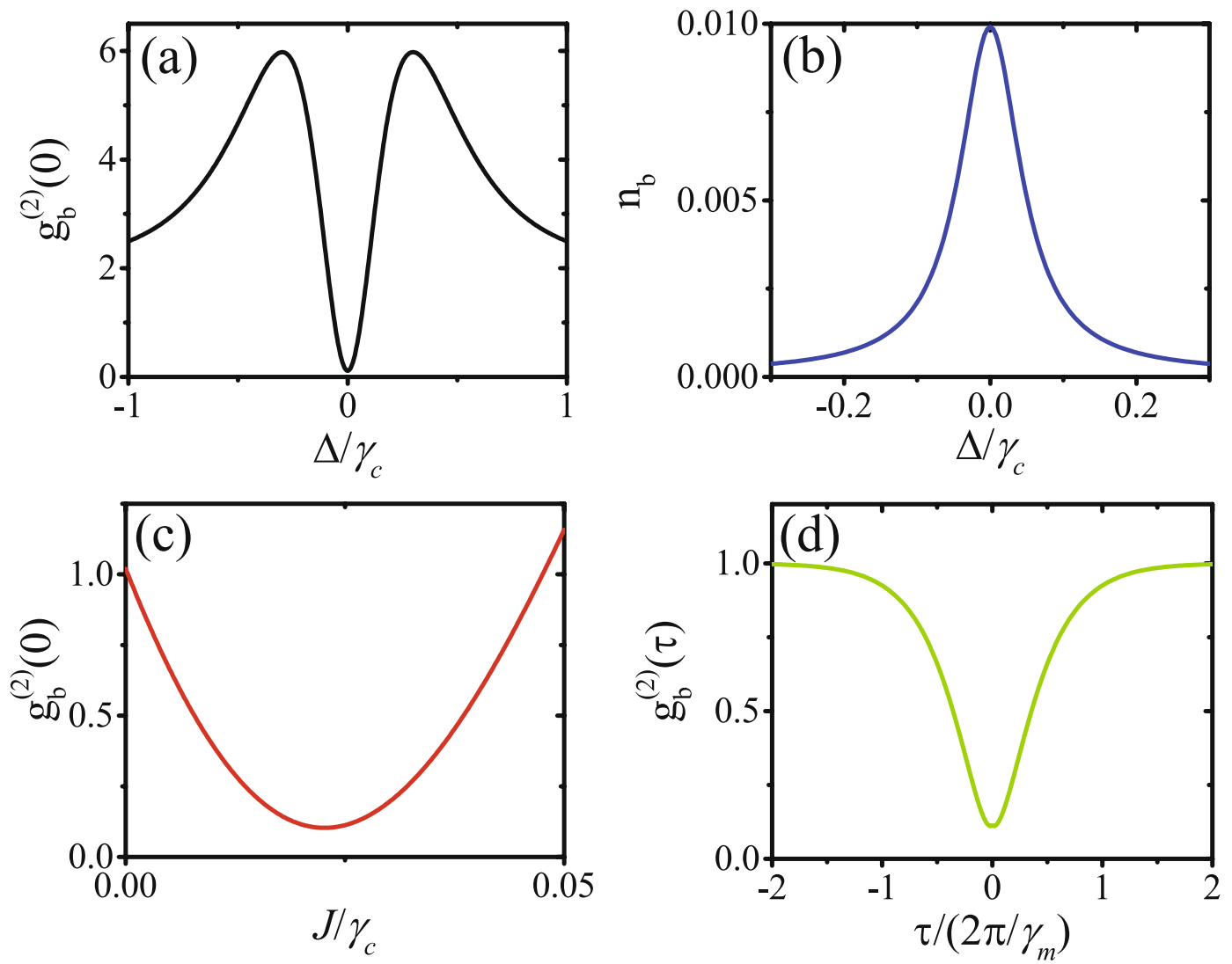

Figure 2. (a) $g_{b}^{(2)}(0)$ is plotted as a function of the detuning $\Delta / \gamma_{c}$ with the effective coupling strength $J=0.025 \gamma_{c} ;(\mathbf{b})$ mean phonon number $n_{b}$ is plotted as a function of $\Delta / \gamma_{c}$ with $J=0.025 \gamma_{c} ;(\mathbf{c}) g_{b}^{(2)}(0)$ is plotted as a function of $J / \gamma_{c}$ with $\Delta=0 ;(\mathbf{d}) g_{b}^{(2)}(\tau)$ is plotted as a function of the normalized time delay $\tau /\left(2 \pi / \gamma_{m}\right)$ with $\Delta=0$ and $J=0.025 \gamma_{c}$. The other parameters are $\varepsilon_{m}=0.005 \gamma_{c}, \varepsilon_{p}=0.01 \gamma_{c}, \theta=\pi, \gamma_{m}=\gamma_{c} / 10$, and $n_{\mathrm{th}}=10^{-4}$.
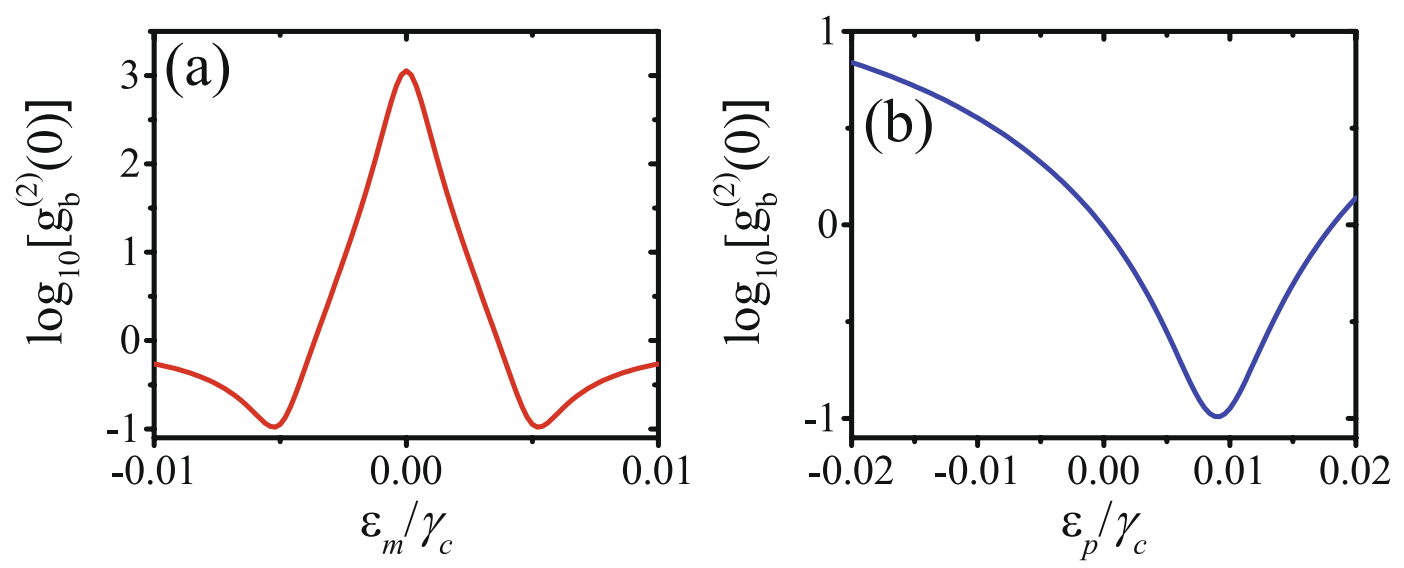

Figure 3. $g_{b}^{(2)}(0)$ is plotted (a) as a function of the mechanical driving strength $\varepsilon_{m} / \gamma_{c}$ with optical driving strength $\varepsilon_{p}=0.01 \gamma_{c}$, (b) as a function of the optical driving strength $\varepsilon_{p} / \gamma_{c}$ with mechanical driving strength $\varepsilon_{m}=0.005 \gamma_{c}$. The other parameters are $\Delta=0, J=0.025 \gamma_{c}, \theta=\pi, \gamma_{m}=\gamma_{c} / 10$, and $n_{\mathrm{th}}=10^{-4}$.

ment with equation $(10) \cdot g_{b}^{(2)}(\tau)$ is plotted as a function of the normalized time delay $\tau /\left(2 \pi / \gamma_{m}\right)$ in Fig. 2 (d) with $\Delta=0, J=0.025 \gamma_{c}$ and $\theta=\pi$. The time duration for strong phonon antibunching is about the lifetime of the phonons.

There are two weak driving fields applied to the system with the driving strengths $\varepsilon_{p}$ and $\varepsilon_{m}$ and they can allow for dynamic control of the phonon statistics by tuning the strengths and the phase difference of driving fields. In Fig. 3 for $\theta=\pi, g_{b}^{(2)}(0)$ is plotted (a) as a function of the mechanical driving strength $\varepsilon_{m} / \gamma_{c}$ with optical driving strength $\varepsilon_{p}=0.01 \gamma_{c}$, (b) as a function of the optical driving strength $\varepsilon_{p} / \gamma_{c}$ with mechanical driving strength 


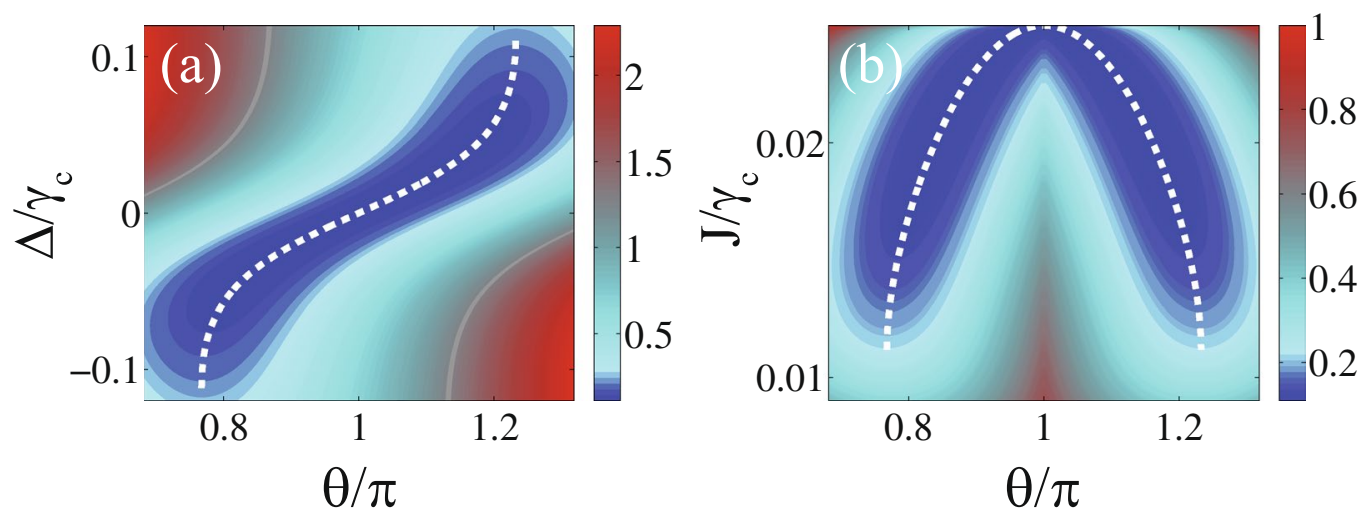

Figure 4. (a) Contour plot of $g_{b}^{(2)}(0)$ as a function of the phase $\theta / \pi$ and the detuning $\Delta / \gamma_{c}$ for effective coupling strength $J$ given in equation (19); (b) contour plot of $g_{b}^{(2)}(0)$ as a function of $\theta / \pi$ and $J / \gamma_{c}$ for $\Delta$ given in equation (17). The white dashed lines refer to equation (11) in (a) and refer to equation (12) in (b). The other parameters are $\varepsilon_{m}=0.005 \gamma_{c}, \varepsilon_{p}=0.01 \gamma_{c}, \gamma_{m}=\gamma_{c} / 10$, and $n_{\mathrm{th}}=10^{-4}$.

$\varepsilon_{m}=0.005 \gamma_{c}$. The minimal $g_{b}^{(2)}(0)$ appears with mechanical driving strength $\varepsilon_{m} \approx \pm 0.005 \gamma_{c}$ in Fig. 3(a) and with optical driving strength $\varepsilon_{p} \approx 0.01 \gamma_{c}$ in Fig. 3(b). These results are consistent with the analytically expression given in equation (10). Moreover, as shown in Fig. 3(a), the phonons exhibit strong bunching as $\varepsilon_{m}=0$ but exhibit strong antibunching as $\varepsilon_{m}=0.005 \gamma_{c}$. These phenomena can be understand as follows: when $\varepsilon_{m}=0$, phonons only can be generated in pairs by the optical driving field, so the phonons exhibit strong bunching; when $\varepsilon_{m} \neq 0$, phonons pairs can be generated in two different ways (by optical driving field or by mechanical driving field), the strong phonon antibunching is induced by the destructive interference between the two different ways for phonon pairs generation when $\varepsilon_{m} \approx \varepsilon_{p} / 2=0.005 \gamma_{c}$. As shown in Fig. 3(b), the phonons exhibit strong antibunching as $\varepsilon_{p}=0.01 \gamma_{c}$ but exhibit bunching as $\varepsilon_{p}>0.02 \gamma_{c}$ or $\varepsilon_{p}<0$. So we can control the phonon statistics dynamically by tuning the strengths of driving fields.

In Fig. 4(a), we show the contour plot of $g_{b}^{(2)}(0)$ as a function of the phase $\theta / \pi$ and the detuning $\Delta / \gamma_{c}$ with the effective coupling strength $J$ given by

$$
J=-\frac{\varepsilon_{m}^{2}}{\varepsilon_{p}} \frac{\gamma_{c}}{\gamma_{m} \cos \theta_{c}+2 \Delta \sin \theta_{c}},
$$

where

$$
\theta_{c}=\pi+\tan ^{-1}\left[\frac{2 \Delta\left(\gamma_{c}-2 \gamma_{m}\right)}{8 \Delta^{2}+\gamma_{c} \gamma_{m}}\right]
$$

In Fig. 4(b), we show the contour plot of $g_{b}^{(2)}(0)$ as a function of $\theta / \pi$ and $J / \gamma_{c}$ with the detuning $\Delta$ given by

$$
\Delta=\frac{\varepsilon_{p} J \gamma_{m} \sin \theta_{c}}{4 \varepsilon_{m}^{2}+2 \varepsilon_{p} J \cos \theta_{c}},
$$

where

$$
\theta_{c}=\left\{\begin{aligned}
\cos ^{-1}\left[-\frac{\varepsilon_{p}^{2} J^{2} \gamma_{m}+2 \varepsilon_{m}^{4} \gamma_{c}}{\varepsilon_{m}^{2} \varepsilon_{p} J\left(2 \gamma_{m}+\gamma_{c}\right)}\right] & \pi / 2<\theta<\pi, \\
2 \pi-\cos ^{-1}\left[-\frac{\varepsilon_{p}^{2} J^{2} \gamma_{m}+2 \varepsilon_{m}^{4} \gamma_{c}}{\varepsilon_{m}^{2} \varepsilon_{p} J\left(2 \gamma_{m}+\gamma_{c}\right)}\right] & \pi<\theta<3 \pi / 2 .
\end{aligned}\right.
$$

The white dashed lines refer to equation (11) in Fig. 4(a) and refer to equation equation (12) in Fig. 4(b). The white dashed lines conform very closely to optimal region (dark blue region) for phonon antibunching. Obviously, the phonon statistic properties are also dependent on the phase difference $\theta$ of the driving fields.

Different from the photon blockade in optical cavities with frequency $10^{14} \mathrm{~Hz}$, where the mean thermal photon number is negligible, the effect of the thermal phonons should be considered in the investigation of phonon blockade in mechanical resonators even with microwave-frequency ${ }^{13}$. In Fig. $5(\mathrm{a}), g_{b}^{(2)}(0)$ is plotted as a function of the mean thermal phonon number $n_{\mathrm{th}}$. One can see that the phonon antibunching becomes weaker with the increase of the the mean thermal phonon number $n_{\mathrm{th}}$. In Fig. 5(b), $g_{b}^{(2)}(0)$ is plotted as a function of the driving strength $\varepsilon_{m} / \gamma_{c}$ with different mean thermal phonon number $n_{\text {th }}$. The optimal phonon blockade can be obtained by properly increasing the driving strengths according to the mean thermal phonon number $n_{\mathrm{th}}$. 

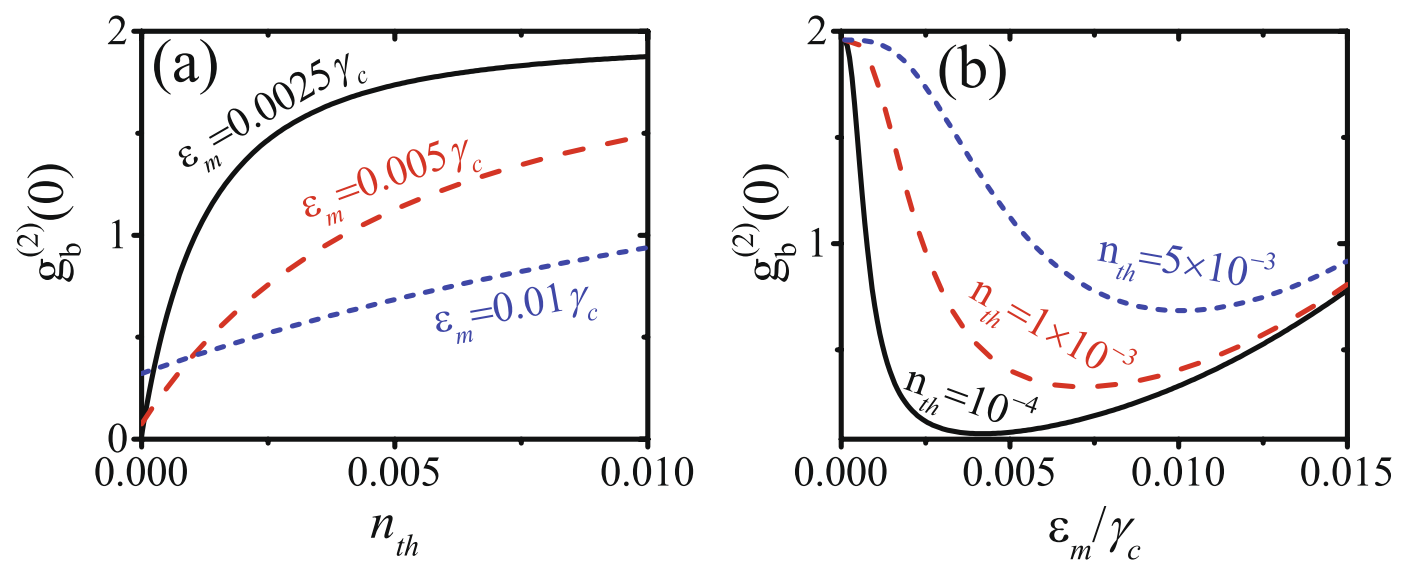

Figure 5. (a) $g_{b}^{(2)}(0)$ is plotted as a function of the mean thermal phonon number $n_{\text {th }}$ with different driving strengths $\varepsilon_{m} / \gamma_{c}$, (b) $g_{b}^{(2)}(0)$ is plotted as a function of the driving strength $\varepsilon_{m} / \gamma_{c}$ with different mean thermal phonon number $n_{\mathrm{th}}$. The other parameters are $\Delta=0, J=0.025 \gamma_{c}, \theta=\pi, \varepsilon_{p}=\varepsilon_{m}^{2} /\left(0.0025 \gamma_{c}\right)$ and $\gamma_{m}=\gamma_{c} / 10$.

\section{Discussion}

In summary, we have investigated the UCPNB in a quadratically coupled optomechanical system. It has been shown that strong phonon antibunching can be observed even with weak effective second-order nonlinear interaction. The optimal conditions for UCPNB were given analytically and they well coincided with the numerical results. Moreover, the phonon statistics can be dynamically controlled by tuning the strengths and the phase difference of external driving fields. The results show that tunable single-phonon sources can be realize in the quadratically coupled optomechanical systems.

Based on the numerical results, we can estimate the experimental parameters for realizing our proposal. For instance, if we take the parameters according to the numerical simulations in ref. ${ }^{35}, \omega_{m} / 2 \pi=225 \mathrm{MHz}, \gamma_{c} / 2 \pi=20$ $\mathrm{MHz}, g / 2 \pi=10 \mathrm{kHz}$, and $\gamma_{m} / 2 \pi=80 \mathrm{kHz}$, then the effective coupling strength $J=0.025 \gamma_{c}$ can be realized with $|\alpha|=50$ when the strength of the strong optical driving field is taken as $\Omega_{L} \approx 27.5 \mathrm{GHz}$. In order to reduce the negative impact of the environment on the phonon statistics, the experiments should be done under low temperature with high mechanical frequency. The mechanical resonators with frequency above $5 \mathrm{GHz}$ have already be realized in many groups ${ }^{45,46}$, and the mean thermal phonon number will be smaller than $10^{-4}$ at a temperature of $25 \mathrm{mK}$ in a dilution refrigerator. So far as we know, the second-order correlation of phonons can not be observed directly. In a recent experiment, the correlations of phonons have been observed indirectly by coupling an auxiliary optical cavity to the mechanical resonator and measuring photon correlations of the output field from the optical cavity ${ }^{47}$.

\section{Methods}

In this section, we will derive the optical conditions for UCPNB analytically with the effective Hamiltonian $H_{\text {eff }}$ given in equation (6), in the limit $T \rightarrow 0$ and the weak driving condition $\left\{\varepsilon_{p}, \varepsilon_{m}\right\} \ll\left\{\gamma_{c}, \gamma_{m}\right\}$. The wave function can be expanded on a Fock state basis as

$$
|\psi\rangle=C_{00}|0,0\rangle+C_{10}|1,0\rangle+C_{01}|0,1\rangle+C_{02}|0,2\rangle+\cdots,
$$

where $|n, m\rangle$ represents the state with $n$ photons and $m$ phonons, and the corresponding coefficient $\left|C_{n m}\right|^{2}$ denotes the occupying probability. In the weak driving condition, i.e. $\left\{\varepsilon_{p}, \varepsilon_{m}\right\} \ll\left\{\gamma_{c}, \gamma_{m}\right\}$, we will have $\left|C_{00}\right| \gg\left\{\left|C_{10}\right|,\left|C_{01}\right|,\left|C_{02}\right|\right\} \gg\left\{\left|C_{11}\right|,\left|C_{02}\right|,\left|C_{12}\right|\right\} \gg \cdots$, so the wave function can be truncated to the one-photon and two-phonon states approximately. Substituting the wave function in equation (19) and the Hamiltonian in equation (6) into the Schrödinger's equation $i d|\psi\rangle / d t=H_{\mathrm{eff}}|\psi\rangle$, then the dynamical equations for the coefficients $C_{n m}$ are shown as

$$
\begin{gathered}
\frac{d}{d t} C_{10}=-\left(\frac{\gamma_{c}}{2}+i 2 \Delta\right) C_{10}-i \varepsilon_{p} C_{00}-i \sqrt{2} J e^{i \theta} C_{02}, \\
\frac{d}{d t} C_{01}=-\left(\frac{\gamma_{m}}{2}+i \Delta\right) C_{01}-i \varepsilon_{m} C_{00}, \\
\frac{d}{d t} C_{02}=-\left(\gamma_{m}+i 2 \Delta\right) C_{02}-i \sqrt{2} \varepsilon_{m} C_{01}-i \sqrt{2} J e^{-i \theta} C_{10} .
\end{gathered}
$$

In the steady state, i.e. $d C_{n m} / d t=0$, the phonon blockade $g_{b}^{(2)}(0) \approx 0$ appears when $C_{02} \approx 0$. Under the condition for phonon blockade, i.e. $C_{02} \approx 0$, the coefficients $C_{10}, C_{01}$ and $C_{00}$ satisfy the linear equations 


$$
\begin{aligned}
& 0=-\left(\frac{\gamma_{c}}{2}+i 2 \Delta\right) C_{10}-i \varepsilon_{p} C_{00}, \\
& 0=-\left(\frac{\gamma_{m}}{2}+i \Delta\right) C_{01}-i \varepsilon_{m} C_{00}, \\
& 0=-i \sqrt{2} \varepsilon_{m} C_{01}-i \sqrt{2} J e^{-i \theta} C_{10} .
\end{aligned}
$$

From equations (23) and (24), $C_{10}$ and $C_{01}$ are given by

$$
\begin{gathered}
C_{10}=\frac{-i 2 \varepsilon_{p}}{\gamma_{c}+i 4 \Delta} C_{00}, \\
C_{01}=\frac{-i 2 \varepsilon_{m}}{\gamma_{m}+i 2 \Delta} C_{00} .
\end{gathered}
$$

Substituting $C_{10}$ and $C_{01}$ into equation (25), we obtain

$$
0=\left(\frac{\varepsilon_{m}^{2}}{\gamma_{m}+i 2 \Delta}+\frac{\varepsilon_{p} J e^{-i \theta}}{\gamma_{c}+i 4 \Delta}\right) C_{00} .
$$

As $\left|C_{00}\right| \approx 1 \neq 0$, then we get the conditions for the optimal parameters $J_{\mathrm{opt}}$ and $\Delta_{\text {opt }}$ as

$$
\begin{gathered}
\varepsilon_{p} J_{\mathrm{opt}}\left(\gamma_{m} \cos \theta+2 \Delta_{\mathrm{opt}} \sin \theta\right)+\varepsilon_{m}^{2} \gamma_{c}=0, \\
\varepsilon_{p} J_{\mathrm{opt}}\left(2 \Delta_{\mathrm{opt}} \cos \theta-\gamma_{m} \sin \theta\right)+4 \varepsilon_{m}^{2} \Delta_{\mathrm{opt}}=0 .
\end{gathered}
$$

The optimal parameters for phonon blockade given in equations (9-13) are obtained by solving the equations (29) and (30).

\section{References}

1. Liu, Y. X. et al. Qubit-induced phonon blockade as a signature of quantum behavior in nanomechanical resonators. Phys. Rev. A 82, 032101 (2010).

2. Kastner, M. A. Artificial Atoms. Phys. Today 46(1), 24 (1993).

3. Imamoglu, A., Schmidt, H., Woods, G. \& Deutsch, M. Strongly Interacting Photons in a Nonlinear Cavity. Phys. Rev. Lett. 79, 1467 (1997).

4. Tong, D. et al. Local Blockade of Rydberg Excitation in an Ultracold Gas. Phys. Rev. Lett. 93, 063001 (2004).

5. Singer, K., Reetz-Lamour, M., Amthor, T., Marcassa, L. G. \& Weidemüller, M. Suppression of Excitation and Spectral Broadening Induced by Interactions in a Cold Gas of Rydberg Atoms. Phys. Rev. Lett. 93, 163001 (2004).

6. Liebisch, T. C., Reinhard, A., Berman, P. R. \& Raithel, G. Atom Counting Statistics in Ensembles of Interacting Rydberg Atoms. Phys. Rev. Lett. 95, 253002 (2005).

7. Vogt, T. et al. Dipole Blockade at Förster Resonances in High Resolution Laser Excitation of Rydberg States of Cesium Atoms. Phys. Rev. Lett. 97, 083003 (2006).

8. Zhang, Z. et al. Phase Modulation in Rydberg Dressed Multi-Wave Mixing processes. Sci. Rep. 5, 10462 (2015).

9. Didier, N., Pugnetti, S., Blanter, Y. M. \& Fazio, R. Detecting phonon blockade with photons. Phys. Rev. B 84, 054503 (2011).

10. Miranowicz, A., Bajer, J., Lambert, N., Liu, Y. X. \& Nori, F. Tunable multiphonon blockade in coupled nanomechanical resonators. Phys. Rev. A 93, 013808 (2016).

11. Wang, X., Miranowicz, A., Li, H. R. \& Nori, F. Method for observing robust and tunable phonon blockade in a nanomechanical resonator coupled to a charge qubit. Phys. Rev. A 93, 063861 (2016).

12. Ramos, T., Sudhir, V., Stannigel, K., Zoller, P. \& Kippenberg, T. J. Nonlinear Quantum Optomechanics via Individual Intrinsic TwoLevel Defects. Phys. Rev. Lett. 110, 193602 (2013).

13. Xu, X. W., Chen, A. X. \& Liu, Y. X. Phonon blockade in a nanomechanical resonator resonantly coupled to a qubit. Phys. Rev. A 94, 063853 (2016).

14. Kippenberg, T. J. \& Vahala, K. J. Cavity Optomechanics: Back-Action at the Mesoscale. Science 321, 1172 (2008).

15. Marquardt, F. \& Girvin, S. M. Optomechanics. Physics 2, 40 (2009).

16. Aspelmeyer, M., Meystre, P. \& Schwab, K. Quantum optomechanics. Phys. Today 65(7), 29 (2012).

17. Aspelmeyer, M., Kippenberg, T. J. \& Marquardt, F. Cavity Optomechanics. Rev. Mod. Phys. 86, 1391 (2014).

18. Metcalfe, M. Applications of cavity optomechanics. Appl. Phys. Rev. 1, 031105 (2014).

19. Seok, H. \& Wright, E. M. Antibunching in an optomechanical oscillator. Phys. Rev. A 95, 053844 (2017).

20. Xie, H., Liao, C. G., Shang, X., Ye, M. Y. \& Lin, X. M. Phonon blockade in a quadratically coupled optomechanical system. Phys. Rev. A 96, 013861 (2017).

21. Liew, T. C. H. \& Savona, V. Single Photons from Coupled Quantum Modes. Phys. Rev. Lett. 104, 183601 (2010).

22. Bamba, M., Imamoglu, A., Carusotto, I. \& Ciuti, C. Origin of strong photon antibunching in weakly nonlinear photonic molecules. Phys. Rev. A 83, 021802(R) (2011).

23. Lemonde, M. A., Didier, N. \& Clerk, A. A. Antibunching and unconventional photon blockade with Gaussian squeezed states. Phys. Rev. A 90, 063824 (2014).

24. Majumdar, A., Bajcsy, M., Rundquist, A. \& Vuckovic, J. Loss-Enabled Sub-Poissonian Light Generation in a Bimodal Nanocavity. Phys. Rev. Lett. 108, 183601 (2012).

25. Kómár, P. et al. Single-photon nonlinearities in two-mode optomechanics. Phys. Rev. A 87, 013839 (2013).

26. Gerace, D. \& Savona, V. Unconventional photon blockade in doubly resonant microcavities with second-order nonlinearity. Phys. Rev. A 89, 031803(R) (2014). 
27. Kyriienko, O., Shelykh, I. A. \& Liew, T. C. H. Tunable single-photon emission from dipolaritons. Phys. Rev. A 90, 033807 (2014).

28. Xu, X. W. \& Li, Y. J. Antibunching photons in a cavity coupled to an optomechanical system. J. Opt. B: At. Mol. Opt. Phys. 46, 035502 (2013).

29. Xu, X. W. \& Li, Y. Strong photon antibunching of symmetric and antisymmetric modes in weakly nonlinear photonic molecules. Phys. Rev. A 90, 033809 (2014).

30. Xu, X. W. \& Li, Y. Tunable photon statistics in weakly nonlinear photonic molecules. Phys. Rev. A 90, 043822 (2014).

31. Kyriienko, O. \& Liew, T. C. H. Triggered single-photon emitters based on stimulated parametric scattering in weakly nonlinear systems. Phys. Rev. A 90, 063805 (2014).

32. Shen, H. Z., Zhou, Y. H. \& Yi, X. X. Tunable photon blockade in coupled semiconductor cavities. Phys. Rev. A 91, 063808 (2015).

33. Zhou, Y. H., Shen, H. Z. \& Yi, X. X. Unconventional photon blockade with second-order nonlinearity. Phys. Rev. A 92, 023838 (2015).

34. Guan, S., Bowen, W. P., Liu, C. \& Duan, Z. Phonon antibunching effect in coupled nonlinear micro/nanomechanical resonator at finite temperature. Europhys. Lett. 119, 58001 (2017)

35. Paraso, T. K. et al. Position-squared coupling in a tunable photonic crystal optomechanical cavity. Phys. Rev. X 5, 041024 (2015).

36. Flowers-Jacobs, N. E. et al. Fiber-cavity-based optomechanical device. Appl. Phys. Lett. 101, 221109 (2012).

37. Thompson, J. D. et al. Strong dispersive coupling of a high-finesse cavity to a micromechanical membrane. Nature 452, 72 (2008).

38. Xu, H., Mason, D., Jiang, L. \& Harris, J. G. E. Topological energy transfer in an optomechanical system with exceptional points. Nature 537, 80 (2016).

39. Xu, H. et al. Observation of optomechanical buckling transitions. Nat. Commun. 8, 14481 (2017).

40. Purdy, T. P. et al. Tunable Cavity Optomechanics with Ultracold Atoms. Phys. Rev. Lett. 105, 133602 (2010).

41. Hill, J. T. N Optics and Wavelength Translation via Cavity-Optomechanics, Ph.D. thesis, California Institute of Technology (2013).

42. Doolin, C. et al. Nonlinear Optomechanics in the Stationary Regime. Phys. Rev. A 89, 053838 (2014).

43. Brawley, G. A. et al. Nonlinear optomechanical measurement of mechanical motion. Nat. Commun. 7, 10988 (2016).

44. Carmichael, H. J. An Open Systems Approach to Quantum Optics, Lecture Notes in Physics Vol. 18 (Springer-Verlag, Berlin, 1993).

45. Fang, K., Matheny, M. M., Luan, X. \& Painter, O. Optical transduction and routing of microwave phonons in cavity-optomechanical circuits. Nature Photon. 10, 489 (2016)

46. Han, X., Zou, C. \& Tang, H. Multimode Strong Coupling in Superconducting Cavity Piezoelectromechanics. Phys. Rev. Lett. 117, 123603 (2016).

47. Cohen, J. D. et al. Phonon counting and intensity interferometry of a nanomechanical resonator. Nature 520, 522 (2015).

\section{Acknowledgements}

This work was supported by the National Natural Science Foundation of China under Grant No.11604096.

\section{Author Contributions}

H.Q.S. and X.W.X. conceived the idea and carried out the calculation. N.H.L. supervised the work. All authors contributed to the interpretation of the work and the preparation of the manuscript.

\section{Additional Information}

Competing Interests: The authors declare that they have no competing interests.

Publisher's note: Springer Nature remains neutral with regard to jurisdictional claims in published maps and institutional affiliations.

Open Access This article is licensed under a Creative Commons Attribution 4.0 International License, which permits use, sharing, adaptation, distribution and reproduction in any medium or format, as long as you give appropriate credit to the original author(s) and the source, provide a link to the Creative Commons license, and indicate if changes were made. The images or other third party material in this article are included in the article's Creative Commons license, unless indicated otherwise in a credit line to the material. If material is not included in the article's Creative Commons license and your intended use is not permitted by statutory regulation or exceeds the permitted use, you will need to obtain permission directly from the copyright holder. To view a copy of this license, visit http://creativecommons.org/licenses/by/4.0/.

(c) The Author(s) 2018 